\title{
ELABORACIÓN DEL DUELO EN FAMILIARES DE LAS VÍCTIMAS DE DESAPARICIÓN FORZADA EN EL DEPARTAMENTO DEL CAQUETÁ
}

\section{MOURNING IN FAMILIES OF VICTIMS OF ENFORCED DISAPPEARANCE IN THE DEPARTMENT OF CAQUETÁ}

\section{ELABORAÇÃO DO LUTO EM FAMILIARES DAS VÍTIMAS DE DESAPARECIMENTO FORÇADO NO DEPARTAMENTO DE CAQUETÁ}

Laura Camila Romero Sáenz

Universidad de la Amazonia

lauraromero2401@gmail.com

Florencia-Colombia

Jhon Sebastián Gómez Valencia

Universidad de la Amazonia gomezvalenciajhonsebastian@gmail.com

Florencia- Colombia

Diagramación

Sindy Catherine Charcas Ibarra

Fotografia portada

Santiago Ardila Acero

Encuentre este artículo en:

http://revistas.uniminuto.edu/index.php/IYD

Para citar este artículo / To cite this article

Romero, L. \& Gómez, J. (2021). Elaboración del duelo en familiares de las

víctimas de desaparición forzada en el departamento del Caquetá. Inclusión \&

Desarrollo, 8 (1), pp 117-130

\section{RESUMEN}

El presente artículo tiene como objetivo comprender la elaboración del duelo en los familiares de las víctimas de desaparición forzada en el Caquetá. Se realizó mediante un enfoque cualitativo de investigación a través del diseño histórico-hermenéutico, en el que se utilizó la entrevista semiestructurada como método de recolección de información. Como resultado se evidenció que la elaboración del duelo se extiende en el tiempo debido al mantenimiento de la esperanza vinculada al regreso de su ser querido con vida, la importancia simbólica del cuerpo para practicar los ritos fúnebres y la necesidad de conocer la verdad en torno a los hechos de la desaparición. A modo de conclusión, se evidencia que contar las historias constituye un elemento sanador y permite conocer las particularidades frente a la elaboración del duelo.

Palabras claves: desaparición forzada, duelo, esperanza, justicia, verdad, víctimas. 


\section{ABSTRACT}

The purpose of this article is to cover the mourning of the families of the victims of enforced disappearance in El Caquetá. It was carried out through a qualitative research approach through the historical-hermeneutic design, in which the semistructured interview was used as a method of information collection. As a result it became clear that the development of mourning extends over time due to the maintenance of hope linked to the return of your loved one alive, the symbolic importance of the body for performing funeral rites and the need to know the truth about the events of disappearance. By way of conclusion, it is evident that telling the stories constitutes a healing element and allows to know the particularities in front of the elaboration of the mourning.

Key words: enforced disappearance, mourning, hope, justice, truth, victims.

\section{SUMÀRIO}

O presente artigo tem como objectivo compreender a elaboração do luto nos familiares das vítimas de desaparecimento forçado no Caquetá. Foi realizada mediante uma abordagem qualitativa de investigação através do desenho históricohermenêutico, no qual se utilizou a entrevista semiestruturada como método de coleta de informações. Como resultado se evidenciou que a elaboração do luto se estende no tempo devido à manutenção da esperança vinculada ao retorno de seu ente querido com vida, a importância simbólica do corpo para praticar os ritos fúnebres e a necessidade de conhecer a verdade em torno dos fatos do desaparecimento. A título de conclusão, é evidente que contar as histórias constitui um elemento curador e permite conhecer as particularidades frente à elaboração do luto.

Palavras chave: desaparecimento forçado, luto, esperança, justiça, verdade, vítimas. 


\section{Introducción}

Colombia ha estado expuesta, desde hace muchos años a diferentes maneras de violencia, presentándose como un mecanismo de represión en cada uno de los sectores sociales en el que se evidencia las diferentes posturas políticas y sociales. Las distintas maneras de la violencia, han evidenciado los hechos atroces del conflicto armado colombiano que en la actualidad vulneran los principales derechos fundamentales del individuo y entre ellos está la desaparición forzada. Considerado como uno de los crímenes de lesa humanidad.

Según el Registro Único de Víctimas (RUV), existe alrededor de 182. 504 víctimas de desaparición forzada en Colombia, siendo 50.048 víctimas directas y 132.456 víctimas indirectas, de las cuales 7.638 víctimas corresponden al departamento del Caquetá, en el que 2.091 son víctimas directas y 5.547 son víctimas indirectas. Por otra parte, "el Registro Nacional de Desaparecidos cuenta con un total de 89.736 casos de personas que han sido reportadas como desaparecidas" (Segura y Ramírez, 2013, p.481). Lo anterior, refleja la incongruencia de cifras en los registros de casos de personas desaparecidas por parte de las diferentes instituciones gubernamentales, en el que se evidencia la verdadera complejidad de la problemática de la desaparición en Colombia.

A parte de la complejidad institucional que refleja la desaparición forzada, también es importante hacer hincapié en la magnitud que implica este fenómeno en los familiares de las personas que han sufrido este flagelo, es decir, en las distintas esferas del individuo hay diversos impactos, como a nivel social: la ruptura de las relaciones interpersonales, el miedo dentro de las comunidades como estrategia de control social, donde se aplicaba esta modalidad para sembrar terror dentro de estas (Maestre,2015), otros de los impactos más significativos es la desconfianza y la fragmentación del tejido social, la cual hace que se silencien las voces de las víctimas (Arroyo,2013).

A nivel familiar: se ha observado que hay una reasignación de roles dentro de la familia, debido a la ausencia de la persona que por lo general, se hace cargo del hogar, y por ello se deben asumir responsabilidades que no poseían anteriormente, en los casos que el padre desaparece, la madre es la encargada de cubrir los gastos del hogar además de liderar los procesos de búsqueda y de los trámites judiciales que esté conlleva (Mejía, 2019).

A nivel individual: miedo, ansiedad, duelos no resueltos, frustración en los proyectos de vida, inestabilidad económica, "acompañado de marcados cambios en los estados de ánimo, provocando dolor profundo, pérdida de interés que perjudica su vida cotidiana, así como la inhibición que impide desarrollar sus actividades" (Castro y Quenoran, 2017, p.85).

\section{Antecedentes}

En numerosas investigaciones se ha indagado sobre el fenómeno de la desaparición forzada alrededor del mundo. Antillón (2018) publicó un diagnóstico sobre las afectaciones psicosociales en torno a los hechos acontecidos el 26 y 27 de Septiembre del 2014 en la ciudad de Iguala-México donde fueron desaparecidos 43 estudiantes indígenas, que denota una serie de estrategias de afrontamiento que le han permitido manejar la situación, aun así, se identifica que estas familias han padecido un tipo de duelo traumático debido a la ausencia de información del paradero de su ser querido, especialmente por la circunstancia violenta que padecieron con anterioridad.

Gancedo (2016), en su estudio: "La herida abierta: el proceso de duelo en familiares de detenidos-desaparecidos por 
el terrorismo de Estado en Uruguay (19731985)" explora las particularidades del proceso y elaboración de duelos en familiares que tiene un ser querido desaparecido a causa del terrorismo en Uruguay, como efecto de la ausencia del cuerpo, se mantiene la esperanza de que el familiar siga con vida, lo cual dificulta la elaboración del duelo y se instaura la incertidumbre como parte de sus vidas. De la misma manera, Arnoso, Arnoso y Pérez (2015), en su estudio "Argentina (1976-1983): impacto y afrontamiento psicosocial", exploran el impacto de la violencia de la última dictadura militar en Argentina en familiares que tiene un ser querido desaparecido y sobrevivientes a las cárceles, el cual refleja las formas de afrontamiento ante situaciones negativas.

En Colombia, uno de los estudios destacados es el informe realizado por el Centro Nacional de Memoria Histórica (CNMH, 2018), denominado "Caquetá: una autopsia sobre la desaparición forzada", no solo hace énfasis en un recuento de la investigación de la Línea de Antropología Forense del $\mathrm{CNMH}$, además es el relato de la experiencia del encargado del equipo de forenses, Helka Quevedo y el diálogo constante con los familiares de las víctimas de desaparición forzada. En primer lugar, el camino que deben de tener los familiares para llegar a la identificación de los cuerpos y una segunda instancia, es la esperanza viva que tienen los familiares de llegar a encontrarlos. Por otro lado, Helka se propone esclarecer en el informe las maneras y las razones de por qué los "desaparecidos aún se pierden", donde se ofrece un amplio panorama del ejercicio de las diversas instituciones que tienen a cargo la investigación de este crimen, identificación de los cuerpos, la verdad, justicia y la reparación.

Así mismo, el CNMH (2014) en su informe "Entre la incertidumbre y el dolor: impactos psicosociales de la desaparición forzada", refleja los impactos psicosociales de la desaparición forzada por cuanto expone la dimensión del sufrimiento y el dolor, de quienes se ven sometidos a tan aberrante experiencia, realizando un ejercicio de memoria a través de testimonios, los cuales permiten comprender la realidad de los familiares y conocer los diferentes contextos: sociales, culturales y políticos del cual construyen. Cabe resaltar, que este delito ocasiona daños indelebles en la salud mental de la persona, del cual se evidencia la incertidumbre, tristeza, angustia que permanecen en el tiempo y ese sentimiento de indolencia por parte de las instituciones encargadas de los procesos investigativos y ante la respuesta que se les ha manifestado a los familiares.

Acosta y Erazo (2018), por su parte, en la investigación titulada "Rememorar más allá del dolor: construcción de narrativas frente al proceso de duelo en situaciones de desaparición forzada", comprenden la narrativa que moviliza los procesos de duelo en mujeres que tienen un familiar desaparecido, desde el construccionismo social, paradigma de la complejidad y la teoría general de los sistemas. Lo cual muestra la resiliencia y la resistencia como emergencia de recursos personales en el proceso de re-significación de la pérdida.

A su vez, Ariza (2016) desde su trabajo de grado "El concepto de duelo en los familiares de los desaparecidos: una revisión de la literatura" aborda las características a nivel conceptual del duelo de los familiares de personas que han sido desaparecidas de manera forzada, violando con tal acto una serie de derechos humanos, dicho concepto ha sido abordado desde la jurisprudencia a nivel internacional y a nivel académico mediante la revisión en diferentes revistas de investigación, todo esto en pro de reivindicar los derechos y fijar el marco conceptual dentro de la reparación integral a las víctimas de este flagelo. 


\section{Marco teórico}

A partir de la temática abordada, existen diferentes autores que definen la desaparición forzada como:

...el arresto, la detención, el secuestro o cualquier otra forma de privación de libertad que sean obra de agentes del Estado o por personas o grupos de personas que actúan con la autorización, el apoyo o la aquiescencia del Estado, seguida de la negativa a reconocer dicha privación de libertad o del ocultamiento de la suerte o el paradero de la persona desaparecida, sustrayéndola a la protección de la ley. (Convención Internacional para la protección de todas las personas contra las desapariciones forzadas, 2006).

Por otra parte, el Centro Nacional de Memoria Histórica (2014) plantea una definición de desaparición forzada desde un enfoque psicosocial, donde la experiencia posee un carácter traumático que lesiona de forma grave el psiquismo individual de los familiares y demás personas cercanas, además de que impacta negativamente en la sociedad en general. Por último, la desaparición es concebida como una forma de violencia política que deforma la ciudadanía que posee un ser humano, la cual viola los derechos que lo constituye a raíz de un perpetrador, que implica tortura, maltrato y demás formas de violencia extrema separando de su contexto y ubicando en un espacio no localizable (Casado, 2017).

En el fenómeno de la desaparición forzada, se presenta un proceso de duelo el cual es concebido como la reacción totalmente natural que se tiene frente a la pérdida de un ser querido, objeto o ante la exposición a un evento totalmente significativo lo cual implica una reacción emocional y comportamental que refleja sufrimiento cuando este vínculo llega a su fin (Meza et al., 2008). A sí mismo, el duelo se constituye como un agujero dentro de la realidad de las personas, a partir de esto se moviliza el ser querido a un plano simbólico y este proceso es necesario dentro de la adaptación que se debe tener frente a la pérdida debido al impacto emocional que se tiene (Acosta y Erazo,2018).

Dentro del contexto de la desaparición forzada no se presentan los elementos necesarios para que el duelo habitual sea llevado a cabo, esto responde a la ausencia del cadáver sin los rituales fúnebres, a ello se le suma de que si no existe el reporte de que la persona ha fallecido y sobre todo de las causas de su deceso (Ospina, Gómez y Echavarría, 2019).

\section{Metodología Diseño}

Se realizó un estudio con enfoque cualitativo, para comprender la vida social de manera holística, a través de los discursos, palabras, textos que determinan un fenómeno social (Herrera, 2017). Esto articula las dimensiones histórica, cultural, socio-política y contextual, y evita que los procesos de significación de los protagonistas vayan a ser "traducidos" por quienes investigan, a partir de esto se buscó "describir, comprender e interpretar los fenómenos, a través de las percepciones y significados producidos por las experiencias de los participantes" (Hernández, Fernández y Baptista, 2014, p.11).

La presente investigación está sustentada en un diseño histórico-hermenéutico y etnográfico el cual busca comprender los fenómenos sociales y de esta manera se realiza una crítica a la tradición metodológica que ha sido utilizada en las ciencias sociales (Pérez, 2011). Se realizó un muestreo intencional no probabilístico, a través de la técnica de bola de nieve que permite el contacto con otros, esta cadena de referencia se constituye por uno o más sujetos que deben responder al mismo 
objeto de estudio (Izquierdo, 2015).

\section{Participantes}

Los participantes fueron ocho personas adultas, dos hombres y seis mujeres, con edades comprendidas entre los 40 y 78 años. Se realizó la investigación en la Unión Peneya, municipio de Montañita; Río Negro, municipio de Puerto Rico; el barrio La Gloria, municipio de Florencia; Portal La Mono, municipio de Belén de los Andaquíes, para un total de 4 municipios del Departamento del Caquetá.

\section{Instrumentos}

Se realizaron entrevistas semiestructuradas, entendidas como "una guía de asuntos o preguntas y el entrevistador tiene la libertad de introducir preguntas adicionales para precisar concepto u obtener mayor información" (Fernández y Baptista, 2014, p.403). En la guía de la entrevista se abordaron 4 dimensiones: dimensión subjetiva/ emocional, dimensión grupal e interpersonal, dimensión sociopolítica/ participativa y finalmente dimensión histórica cultural; es importante aclarar que dichas dimensiones responden exclusivamente como medio de recolección de información, más no las delimita como variables de análisis. Para el desarrollo del Mapeo colectivo, se trabajó a partir de la metodología de Risler y Ares (2018), ellos afirman que es un "proceso de creación que subvierte el lugar de enunciación para desafiar los relatos dominantes sobre los territorios, a partir de los saberes y experiencias cotidianas de los participantes. Sobre un soporte gráfico y visual se visibilizan las problemáticas" (p. 2).

Para poder realizar de manera ética la presente investigación se hizo uso del consentimiento informado, que permitió la validación del siguiente paso a seguir de la misma, dicho consentimiento es concebido como un proceso necesario en la relación investigador-sujeto que debe estar plasmado dentro de la investigación, para esto es necesario que el sujeto otorgue el consentimiento de manera libre, voluntaria y consciente a partir de la información verídica que se le ha comunicado (Pérez y Moreno, 2019).

\section{Procedimiento}

Los encuentros con las comunidades fueron de carácter integrador y colectivo se trabajó en cuatro encuentros desde la comodidad de sus hogares, generándose un ambiente de confianza y fraternidad con facilidad, con esto se narra no solo los hechos dolorosos sino también anécdotas familiares y territoriales. Como primer momento, se presentó la propuesta metodológica a las comunidades, de esta manera se agendaron los próximos encuentros. En el segundo encuentro, se elaboró la producción de mensajes propios que parten de la construcción de las narrativas, el cual amplió el horizonte de comprensión de los hechos y del impacto que estos han tenido tanto a nivel personal como a nivel familiar.

Como tercer encuentro, se realizó el mapeo colectivo como parte de la producción de mensajes propios e implicó un proceso pedagógico, comunitario y de reconfiguración de los imaginarios del territorio. La sistematización de la información se realizó mediante la transcripción de las entrevistas grabadas, permitiendo denotar la forma en como la esperanza se presenta como una paradoja dentro del proceso que supondría la elaboración del duelo pero que en realidad se extiende interminablemente. En el último encuentro se presentó la construcción de sus relatos y se deja clara la posibilidad de realizar algunas modificaciones en las Historias de Vida. 


\section{Resultados}

Los resultados construidos en la presente investigación responden a tres categorías de análisis de los datos; Como producto de las narraciones se describen los procesos de duelo y el mantenimiento de la esperanza, el cuerpo como elemento simbólico, por último, la lucha constante de la verdad y justicia. Finalmente, se logró consolidar una organización de la sociedad civil en aras de continuar el acompañamiento psicosocial con los familiares y fortalecer los lazos de confianza a nivel comunitario. A continuación se presentan los datos expuestos en cada categoría:

\section{Categoría}

\section{El mantenimiento de la esperanza en el proceso de duelo}

Algunos familiares aguardan la esperanza de que su ser querido se encuentre con vida, el cual les impide continuar con el proceso de duelo, ya que este se encuentra suspendido por la incertidumbre, en este sentido, saber lo que sucedió con su ser querido permitirá culminar de manera positiva su proceso de duelo.

La configuración de las narrativas construidas con los familiares de las personas dadas por desaparecidas permite denotar la esperanza, lo cual refleja en el familiar la negación frente a la muerte. Lo anterior se refleja a continuación:

(...) "Yo no pierdo la esperanza de que mi hermano esté vivo" (Señora R, La Mono). "Aunque mi hijo Regulo lleva ocho años desaparecido, no pierdo la esperanza de que esté vivo y algún día regresará." (Señora F1, La Mono).

Se evidencia que, a pesar de algunos rastros o información importante para los procesos de búsqueda por parte de los familiares, esto queda corto, pues hasta no saber la realidad del paradero de la persona, la esperanza se alimenta con la más mínima información que personas conocidas suministren.

"A pesar de esto nos comentaron sobre ciertos lugares, buscamos en el río, después entre Maracaibo y Santa Isabel, pero no encontramos nada, lo único que quiero saber es dónde está mi hijo" (Señora A, Rio Negro). "Por nuestra parte aún seguimos a la espera... Quizás ahora con las tecnologías, los teléfonos, uno investigando, alguien diga "yo lo distingo" (Señora L1, Rio Negro).

Frente a la intriga que causa el desconocimiento del paradero del desaparecido, se genera una paradoja en las familias refiriéndose a este como un ser que no existe en el mundo material pero que anhelan el regreso con vida, el recuerdo tiene origen en la ausencia y la misma muerte pero se anhela su existencia y pronta presencia, por lo tanto, se genera un gran sufrimiento.

"Desde ese día me perdí yo también, en la desesperación y la eterna angustia de pensar dónde está, quién la tiene. De vivir entre la esperanza de encontrarla viva o de encontrarla muerta" (Señora F2, Florencia). "Él era muy cariñoso conmigo, me quería mucho. La esperanza que tengo es que de pronto lo tengan vivo en alguna parte" (Señor $P, L a$ mono).

Lo anterior, refleja la necesidad de encontrar su ser querido ya que su recuerdo se impregna en los familiares, el cual causa una eterna angustia que culmina con la aparición de los restos óseos de su familiar.

\section{El deseo de recuperar el cuerpo}

El valor simbólico del cuerpo ausente en el caso de la desaparición forzada ha sido una acción que mitiga el dolor y/o impacto, 
convirtiéndose en una práctica que permite conservar la existencia del ser querido en el mundo real. De esta manera, los familiares luchan constantemente por conocer el paradero de sus seres queridos, dando final al sufrimiento que genera la incertidumbre de la desaparición, el cual se le atribuye la responsabilidad al Estado de los procesos de búsqueda.

"Quiero que el Estado me diga: ¡Acá están los restos de su hija! Y así poder darle sepultura y enterrar también esta eterna zozobra, este estado de pena que me acompaña” (F2, Florencia).

Las oraciones, misas y otros rituales se convierten en un medio sanador, tranquilizador y de seguridad frente a la pérdida, el cual refleja el deseo de recuperar el cuerpo y realizar un acto funerario digno, al recuperar el cuerpo se permite cerrar la brecha de dudas e incertidumbre que los ha acompañado por años.

"Estamos ahorita luchando porque entreguen el cuerpo de mi esposo, para poder irle a rezar y dejarle una flor o al menos ir $y$ visitarlo; saber que está ahí, lo acompañamos con nuestra oración" (Señora I, Unión Peneya).

El reconocimiento de cada una de las acciones emprendidas a la búsqueda debido a la desesperanza que padecen los familiares, reflejan el valor simbólico referente al cuerpo y de encontrar sus restos óseos, como es el caso de la elaboración onírica que trasladado a la realidad buscan materializarlo.

"La Fiscalía fue con mi marido a buscarla a Puerto Londoño, porque yo había soñado con ella y me decía que en la cepa de una palma la habían enterrado, pero fueron y no encontraron nada" (Señora F2, Unión Peneya).

\section{Verdad y Justicia}

En el momento en el que se evidencia que un familiar desaparece, inicia un proceso de búsqueda que implica la exigencia de los derechos fundamentales tanto de la persona que está desaparecida como del familiar. Durante las narraciones de los entrevistados se evidencia el conocimiento que tienen acerca de los procedimientos legales que impliquen ser reconocidos como víctimas y lo que conlleva la firma del acuerdo de paz. Lo anterior supone para las víctimas el acceso a la verdad, justicia, reparación y no repetición, todo esto enfocado en la identificación de su ser querido por parte de quienes perpetraron el hecho.

"Sin embargo, no se ha llegado el día en que me diga, ya estamos en el punto cinco, que escuche: "Ya le vamos a entregar a su hijo" no, no se ha llegado ese día y sigo esperando" (Señora L2, Unión Peneya).

"Eulises sigue cumpliendo años. En silencio. Y con mi hermano Ronaldo sabemos que ya es la hora de saber la verdad" (Señora $R$, La Mono).

Para los familiares la reparación y ayudas económicas suponen un factor de menor importancia frente a los casos de desaparición, puesto que la lucha constante por la exigibilidad de sus derechos ha sido: que se haga justicia para saber así la verdad de los hechos.

"El Estado a mí me ha ayudado: con mercados y ayuda humanitaria, pero eso no basta. Acá no hay justicia hasta que no aparezca mi hija" (Señora F2, Florencia).

Asociación de Familiares y Víctimas de Desaparición Forzada en el Caquetá -FAVIDESC

Durante el desarrollo de la investigación se logró identificar fortalezas hacia el interior 
de cada comunidad, a fin de generar vínculos tendientes a la creación de organizaciones locales de víctimas de desaparición forzada en el departamento. Por lo anterior, FAVIDESC tiene como objetivo promover el ejercicio de memoria histórica en búsqueda de la verdad, justicia y reparación en la consolidación de la paz frente a la desaparición forzada y otros hechos victimizantes asociados.

Por último, debido a la sistematización de los datos durante la investigación y la consolidación de la Asociación; en colaboración con su equipo técnico se logró la publicación del informe" narrativo titulado "Ausencias Presentes" en el cual se evidencia de manera directa la realidad de las familias víctimas de desaparición forzada en el departamento, desde un modelo psicosocial. Dicho informe es el primero entregado a la Unidad de Búsqueda de Personas dadas por Desaparecidas (UBPD) en el Caquetá.

\section{Discusión}

Entre los hallazgos encontrados en el presente artículo, resalta tres categorías los cuales se desarrollarán en la presente discusión. El primero de ellos habla acerca del mantenimiento de la esperanza en el proceso de duelo, que refleja un hecho de negación frente a la posible muerte de su ser querido, el cual anhelan el regreso de su familiar y esto se refuerza con el suministro de información o rastros por parte de personas conocidas acerca del paradero del ser desaparecido. Como lo mencionan otros autores, Ardila (2016) plantea que la esperanza de vida representa la ilusión de recibir buenas noticias, especialmente de que su familiar se encuentre con vida, del cual aviva tal ilusión con pensamientos positivos.
De igual forma, Antillo (2018) plantea que la ausencia es concebida de forma transitoria manteniéndose latente la esperanza que el familiar ausente se encuentre con vida, sumándole los comentarios de terceros quienes afirman que el ausente está vivo. Además, la imposibilidad de verificar los testimonios por parte de los perpetradores provoca que la incertidumbre se siga manteniendo, en especial por la ausencia del cuerpo lo que fija una luz de esperanza y vida (CNMH, 2016).

En relación con la segunda categoría distintos estudios están de acuerdo con que la ausencia del cuerpo ha denotado un valor simbólico durante los rituales, como bien lo menciona Arango (2019) "La ausencia de un cuerpo imposibilita la realización de los rituales fúnebres que se realizan en torno a la pérdida de una persona" (p.151). Es por eso que las acciones o actos simbólicos como rezar, realizar cristiana sepultura y visitarlos en el cementerio, demuestran las prácticas, tradiciones y rituales propios de cada cultura. Ortiz, Romero y Blum (2017) señalan que la ausencia del cuerpo cancela la posibilidad de realizar aquellos actos fúnebres, el cual deja a los familiares en la incertidumbre, entre la ausencia y la presencia.

Lo anterior, denota la importancia de la ubicación, exhumación y entrega de los restos óseos a los familiares para que estos a partir de sus prácticas culturales realizan los ritos que crean pertinentes, lo cual determina la pérdida definitiva de su familiar y así permitir que se elabore de forma adecuada el duelo. Partiendo de lo anterior, Zorio (2011) plantea que:

Son precisamente los ritos y las creencias las herramientas disponibles en la cultura para confrontar esa ausencia y es por esto que son esenciales en los procesos de duelo. Por ejemplo, a través de ritos y creencias

\footnotetext{
1 Informe narrativo publicado gracias al apoyo financiero de América Solidaria y de la Comisión Internacional de Personas Desaparecidas (ICMP) con fondos de la Unión Europea.
} 
constatamos individual y colectivamente que los cadáveres no son desechos mortales sin valor social, cultural y/o subjetivo; todo lo contrario, conservan para los dolientes el valor del objeto amado, al cual los ligan vínculos amorosos y libidinales (p.256).

Finalmente, la tercera y última categoría referente a la verdad, justicia y reparación, los familiares señalanquees laobligación delEstado proporcionarles la verdad acerca de los hechos ocurridos y de hacer justicia frente a los actores que perpetraron tal delito de lesa humanidad, puesto que al estar en organizaciones de la sociedad civil les permitirá vincular a los procesos judiciales, el fortalecimiento de sus habilidades, el empoderamiento de una historia y sin lugar a dudas, desde la institucionalidad deben responder ante la dimensión de la reparación y no repetición. En torno a este aspecto cabe resaltar que el Estado tiene el deber de emprender acciones de búsqueda tal y como lo plantea el CNMH (2018) para así dar respuesta a las solicitudes y exigencias en torno a las necesidades de las víctimas. Además, es el compromiso con los familiares referentes a la justicia y el saber la verdad.

\section{Conclusiones}

En conclusión, la apertura de la palabra mediante la construcción de las narrativas se convierte en un proceso significativo, en la medida que se revela y se comparte información valiosa, íntima en un contexto de acción sin daño, lo cual permite develar el camino de los silencios, de la represión estatal e insurgente, pues el contar, refleja en cada uno de los familiares tranquilidad, paz, seguridad y mayormente esperanza. Además, logró evidenciar que el acto de contar las historias se convierte en un medio sanador, el cual permite que la "víctima" se reconozca dentro de un grupo de personas que han padecido eventos similares.
Esto último, es evidente en las historias de vida de cada participante en el estudio, la mención de su ser querido está acompañada de esperanza, pero a su vez se presenta una paradoja ya que lo esperan vivo o esperan sus restos óseos y así poder practicar los rituales fúnebres propios de una pérdida. Al practicar los ritos se permite culminar la incertidumbre y el sufrimiento acerca del paradero de su familiar y trascienden a elaborar el duelo de manera adecuada.

A pesar de lo anterior, la necesidad constante de conocer la verdad de los hechos ocurridos, determina en gran medida la lucha constante por vincularse a ciertas instituciones y/o participar en procesos que les permita estar al tanto de acontecimientos y/o políticas de gran relevancia en cada uno de sus casos, permitiéndoles reclamar constantemente los derechos que se les fueron violentados en dichos actos colmados de barbarie.

\section{Declaraciones}

Gracias a cada uno de los familiares que permitieron abrir la puerta de su corazón y contarnos sus historias en medio del dolor y la incertidumbre, y sobre todo en medio de la esperanza. Gracias al equipo investigador liderado por Carlos Enrique Zapata Bohorquez y Esteban Sossa Salvarezza como asesor externo, de igual manera a los integrantes del semillero "Tejidos de Paz" adscrito al programa de Psicología de la Universidad de la Amazonia. Gracias a nuestra alma mater, por la aprobación y financiamiento.

Agradecimientos a América Solidaria Colombia, por su financiamiento y hacer posible la publicación del informe narrativo "Ausencias Presentes". De igual forma, a la Comisión Internacional sobre Personas Desaparecidas (ICMP) con fondos de la Unión Europea por confiar en el trabajo de FAVIDESC y permitir 
dar un gran paso con una entrega digna del informe a la Unidad de Búsqueda de Personas dadas por Desaparecidas (UBPD). A su vez, agradecimientos al equipo técnico FAVIDESC por el apoyo y el acompañamiento psicosocial constante a cada uno de los familiares que participaron en este proceso.

El proyecto de investigación presentado por el presente artículo fue aprobado por el comité de ética de la Universidad de la Amazonia y los participantes que hicieron parte de dicha investigación firmaron los consentimientos informados, todo el proceso metodológico y de análisis es acorde a lo dispuesto en el capítulo VII de la Ley 1090 del 2006. Por último, el presente artículo no presenta conflicto de intereses. 


\section{Referencias bibliográficas}

Acosta, M. y Erazo, L. (2018). Rememorar más allá del dolor: Construcción de narrativas frente al proceso de duelo en situaciones de desaparición forzada (Tesis de pregrado). Universidad Santo Tomás, Villavicencio. Recuperado de https://repository.usta.edu.co/ bitstream/handle/11634/15472/2018merelynacosta. pdf?sequence=9\&isAllowed=y

Antillón Najlis, X. (2018). Yo sólo quería que amaneciera. Impactos psicosociales del Caso Ayotzinapa. Ciudad de México: Fundar, Centro de Análisis e Investigación AC Obtenido en: http://fundar. org. mx/mexico/pdf/InformeAyotziFin. pdf el, 11(6), 2018. Recuperado de http:// fundar.org.mx/mexico/pdf/InformeAyotziFin.pdf

Arango Serna, K. T. (2019). Duelo en familiares de víctimas de desaparición forzada que participaron en el proceso de excavación de la escombrera, en la comuna 13 de Medellín. Recuperado de http://bibliotecadigital.udea.edu.co/bitstream/10495/16341/1/ ArangoKelly_2019_DueloFamiliaresVictimas.pdf

Ardila Rincón, L. F. (2016). Significados y emociones de una madre que ha vivido la desaparición forzada de uno de sus hijos: estudio de caso.Recuperado dehttp://bibliotecadigital.iue.edu. co/jspui/bitstream/20.500.12717/96/1/iue_rep_pre_psi_ardila_2016_desparici\%C3\%B3n_ forzada.pdf

Ariza Galindo, H. (2016). El concepto de duelo en los familiares de los desaparecidos: una revisión de la literatura (Doctoral dissertation, Universidad del Rosario). Recuperado https:// repository.urosario.edu.co/flexpaper/handle/10336/12708/Tesis\%20Psicologia\%20V5. pdf?sequence=1\&isAllowed=y

Arnoso, M., Arnoso, A., \& Pérez, P. (2015). Argentina (1976-1983): Impacto y afrontamiento psicosocial. Universitas Psychological, 14(3), 833-842. Recuperado de: http://dx.doi. org/10.11144/Javeriana.upsy14-3.aiap

Arroyo, J. (2013). Impacto psicosocial del conflicto armado en familias víctimas de Buenaventura (Estudio detrescasosenBuenaventura)(tesisdepregrado). UniversidaddelValle, Cali, Colombia. Recuperado de: https://bibliotecadigital.univalle.edu.co/bitstream/handle/10893/10620/CB0503492. pdf; jsessionid=7CBC36A495488ECA9B0C32C1461F0BDA?sequence=1

Casado, D. (2017). La sangre de la desaparición forzada: de la violencia a la filiación a través de la sangre. Sociología y tecnología. Sociología y tecnociencia: Revista digital de sociología del sistema tecnocientífico, 7(1), 81-105. Recuperado de https://dialnet.unirioja. es/servlet/articulo?codigo $=5910290$

Castro, E. y Quenoran, Y. (2018). Impacto psicosocial del conflicto armado en familias víctimas de la desaparición forzada. Revista UNIMAR, 12-12. Recuperado de: http://editorial. umariana.edu.co/revistas/index.php/unimar/article/view/1602

Centro Nacional de Memoria Histórica (2018), Desaparición forzada. Balance de la contribución del CNMH al esclarecimiento histórico, Bogotá, CNMH. Recuperado de http://centrodememoriahistorica.gov.co/wp-content/uploads/2020/01/BALANCE_ DESAPARICION_FORZADA.pdf

Centro Nacional de Memoria Histórica (2018), Caquetá: una autopsia sobre la desaparición 
forzada, Bogotá, CNMH.Recuperado Recuperado de http://centrodememoriahistorica.gov. co/wp-content/uploads/2020/02/caqueta_una-autopsia-sobre-la-desaparicion-forzada.pdf Centro Nacional de Memoria Histórica (2016), Hasta encontrarlos. El drama de la desaparición forzada en Colombia, Bogotá, CNMH. Recuperado de https://centrodememoriahistorica. gov.co/descargas/informes2016/hasta-encontrarlos/hasta-encontrarlos-drama-de-ladesaparicion-forzada-en-colombia.pdf

Centro Nacional de Memoria Histórica(2014). Desaparición forzada Tomo III: Entre la incertidumbre y el dolor: impactos psicosociales de la desaparición forzada. Bogotá: Imprenta Nacional. Recuperado de http://www.centrodememoriahistorica.gov.co/descargas/ informes2014/desaparicion-forzada/Tomo-III.pdf

Convención Internacional para la protección de todas las personas contra las desapariciones forzadas, 20 de Diciembre, 2006, http://comisiondebusqueda.gov.co/images/PDF/ convencion_internacional.pdf

Fernandez, C., y Baptista, P. (2014). Metodología de la investigación Roberto Hernandez Sampieri. México: Mcgraw-HILL / Interamericana Editores, S.A. De C.V. Recuperado de: http://observatorio.epacartagena.gov.co/wp-content/uploads/2017/08/metodologia-de-lainvestigacion-sexta-edicion.compressed.pdf

Gancedo, S. (2016). La herida abierta: el proceso de duelo en familiares de detenidosdesaparecidos por el terrorismo de Estado en Uruguay (1973-1985). Recuperado de https:// sifp.psico.edu.uy/sites/default/files/Trabajos\%20finales/\%20Archivos/gancedo_tfg-proceso_ de_duelo_en_familiares_desaparecidos.pdf

Hernández Sampieri, R., Fernández Collado, C., Y Baptista Lucio, M. (2014). Metodología de la investigación. $6^{a}$ edic. México: Editorial McGraw Hill, Interamericana Editores, S.A. de C.V. Recuperado de https://www.uca.ac.cr/wp-content/uploads/2017/10/Investigacion.pdf

Herrera, J. (2017). La investigación cualitativa. Recuperado de: http://biblioteca.udgvirtual.udg. mx:8080/jspui/bitstream/123456789/1167/1/La\%20investigaci\%C3\%B3n\%20cualitativa.pdf Izquierdo, G. M. (2015). Informantes y muestreo en investigación cualitativa. Investigaciones Andina, 17(30), 1148-1150.Recuperado de: https://www.redalyc.org/pdf/2390/239035878001. pdf

Maestre-Sánchez, W. (2015). La desaparición forzada en Colombia: Agendas pendientes de un problema no resuelto. Vis luris. Revista de derecho y ciencias sociales, 121-144.. Recuperado de https://revistas.usergioarboleda.edu.co/index.php/visiuris/article/view/979 Mejia, A. K. C. (2019). Protocolo de atención psicosocial a víctimas indirectas de desaparición del estado de Morelos.(Tesis de Maestria). Universidad Autónoma del Estado de Morelos, Cuernavaca, México. Recuperado de http://riaa2.uaem.mx/xmlui/bitstream/ handle/20.500.12055/737/COMAJN05T.pdf?sequence=1

Meza, E., García, S ., Torres , A ., Castillo, L., Sauri., S, y Martínez, B (2008). El proceso del duelo. Un mecanismo humano para el manejo de las pérdidas emocionales. Revista de Especialidades Médico-Quirúrgicas, 13(1),28-31.Recuperado en https://www.redalyc.org/ articulo. oa?id=473/47316103007

Ortiz, L. M. Romero, M. P. y Blum, B. (2017). Las particularidades del Duelo en los niños ante 
la desaparición de un ser querido. Revista Chilena de Psicoanálisis 33(1), 1-156. Recuperado de https://www.apch.cl/2018/revista/33_1_2017/Ortiz.pdf

Ospina Varela, B. S., Gómez David, L., y Echavarría Saldarriaga, L. E. (2019). Significación del duelo en víctimas de desaparición forzada (Doctoral dissertation, Corporación Universitaria Minuto de Dios). Recuperado de https://repository.uniminuto.edu/bitstream/ handle/10656/10713/T.P_OspinaVarelaBrayanStiv_2019.pdf?sequence=1\&isAllowed=y

Pérez-Cano HJ, Cesar-Moreno BM. Aspectos históricos del consentimiento informado y su aplicación actual. Rev Med MD. 2019;10.11(3):229-234. Recuperado de https://www. medigraphic.com/pdfs/revmed/md-2019/md193f.pdf

Pérez, D. A. Á. (2011). La hermenéutica y los métodos de investigación en ciencias sociales. Estudios de filosofía, (44), 9-37. Recuperado de: http://www.scielo.org.co/pdf/ef/n44/n44a02. pdf

Risler, J., \& Ares, P. (2018). Manual de mapeo colectivo: recursos cartográficos críticos para procesos territoriales de creación colaborativa Buenos Aires: Tinta Limón, 2013. Recuperado de: http://sedici.unlp.edu.ar/bitstream/handle/10915/93167/.pdf?sequence=1

Segura, A., \& Ramírez, D. (2013). Comportamiento del fenómeno de la desaparición, Colombia, 2013. Forensis, 15(1), 475-514.Recuperado de: https://www.medicinalegal.gov. co/documents/20143/49517/Desaparecidos..pdf

Zorio, S. (2011). El dolor por un muerto-vivo. Una lectura freudiana del duelo en los casos de desaparición forzada. Desde el Jardín de Freud, (11), 251-266. Recuperado de https:// revistas.unal.edu.co/index.php/jardin/article/view/27261/27536 